\title{
Simultaneous Z-contrast/phase contrast imaging of ferroelectric thin films
}

\author{
M.F. Chisholm*, H.N. Lee*, W. Luo**** and S.T. Pantelides**** \\ * Materials Science and Technology Division, Oak Ridge National Laboratory, Oak Ridge, TN \\ ** Department of Physics and Astronomy, Vanderbilt University, Nashville, TN
}

One less obvious result of aberration-correction in a scanning transmission electron microscope (STEM) was the improvement in bright field (BF) STEM imaging. It has long been understood that STEM BF imaging can be equivalent to TEM BF imaging via the principle of reciprocity [1], with the STEM probe-forming aperture equivalent to the TEM objective aperture and the STEM collector aperture equivalent to the TEM condenser aperture. However, the conditions necessary for coherent phase contrast imaging required that the STEM collector aperture had to be much smaller than the objective aperture. Thus, only a small fraction of the incident probe current could be used to form the image. As a result, STEM phase contrast images were extremely noisy and not often used. The situation is vastly improved after aberration correction. By reciprocity, the BF image is now equivalent to an aberration-corrected BF TEM image. The much larger flat-phase region produced by aberration correction allows the use of a collector aperture enlarged an order of magnitude without significant loss of coherence, giving two orders of magnitude increased signal. The resulting STEM BF image is now of high quality and is available simultaneously with the annular dark field image with pixel-to-pixel correlation. An example of the simultaneous phasecontrast and Z-contrast imaging of an $<110>$ projection of a $\mathrm{SrTiO}_{3}\{111\} / \mathrm{Al}_{2} \mathrm{O}_{3}\{001\}$ interface is shown in Figure 1. The phase contrast images contain quantitative information on the composition of light columns such as oxygen, just as in aberration-corrected TEM [2,3].

Recently it has been shown that the cation-oxygen dipoles near domain walls in ferroelectrics could be imaged using the negative spherical-aberration technique in an aberration corrected TEM [4]. Before that, Tian et al. [5] showed that conventional phase contrast imaging could be used measure the relative displacement of cations to anions in $\mathrm{BaTiO}_{3}$. Here we have used simultaneous $\mathrm{Z}$ contrast/phase contrast imaging to reveal the 0.1 to $0.4 \AA$ anion and cation displacements that give rise to the electric polarization in ferroelectric oxide thin films. We have used this real-space probe to investigate the local variation of polarization not only in the perfect lattice but also near defects and interfaces. Figure $2 \mathrm{a}$ is a phase contrast image of the interfacial region in a $\mathrm{Pb}(\mathrm{Zr}, \mathrm{Ti}) \mathrm{O}_{3}(\mathrm{PZT})$ film on a thin $\mathrm{SrRuO}_{3}(\mathrm{SRO})$ metallic oxide electrode layer on a $\mathrm{SrTiO}_{3}$ (STO) substrate. Figure 2b is a plot of the average displacements of the oxygen and $\mathrm{Ti} / \mathrm{Zr}$ columns from the centrosymmetric position as a function of distance from the PZT/SRO interface. We see that the displacements in the PZT layer are relatively constant right up to the interface with SRO and are what one expects for bulk polarization. The displacements are seen to continue in the SRO layer. The atoms are displaced in the same direction as in the PZT layer but the magnitude of the displacements decrease across the layer and are zero at the SRO/STO interface. It appears that the bound charges are being screened within the SRO electrode. Density functional theory was used to calculate the expected atomic distortions at the PTO/SRO interface using a supercell geometry. Figure 2c is a plot of the calculated distortions across the SRO and PTO layers that show good agreement with experiment. Adjustments to the structure that improve the match between theory and experiment will be discussed [6]. 


\section{References}

[1] J.M. Cowley, Appl.Phys. Lett. 15 (1969) 58.

[2] C.-L. Jia, Science 303 (2004) 2001.

[3] C.-L. Jia, M. Lentzen, and K. Urban, Science 299 (2003) 870.

[4] C.-L. Jia, et al., Nature Materials 7 (2008) 57.

[5] W. Tian, et al., in Ferroelectric Thin Films IX, Vol. 655, Materials Research Society, Warrendale, 2000, pp. CC7.8.1-6.

[6] This research was sponsored by the Division of Materials Sciences and Engineering, U.S. Department of Energy.
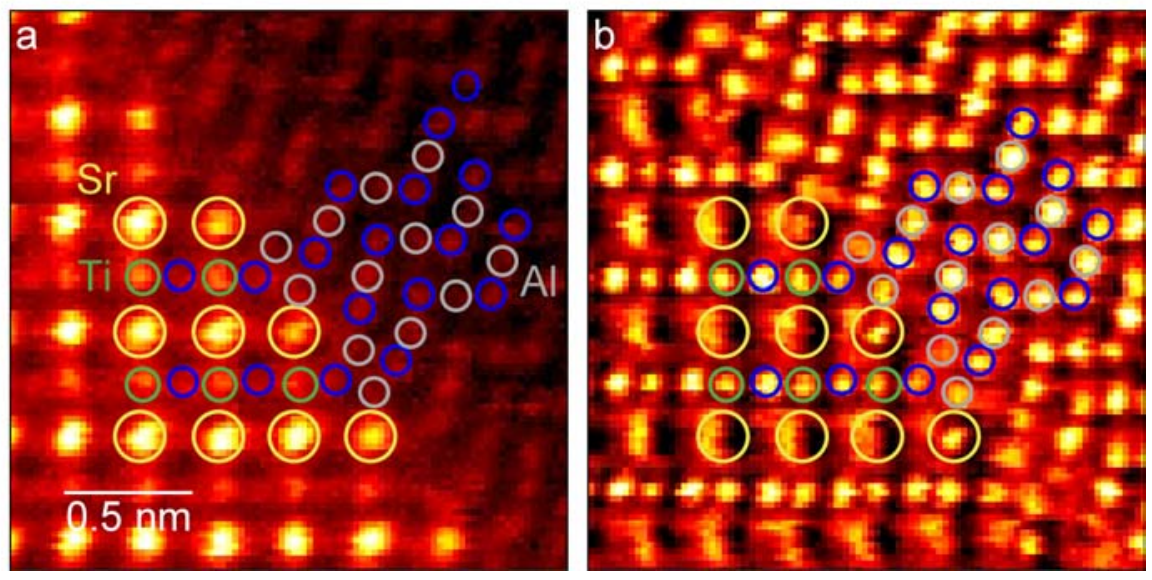

FIG. 1 Comparison of coherent and incoherent imaging of a $\mathrm{SrTiO}_{3} / \mathrm{Al}_{2} \mathrm{O}_{3}$ interface in the $<110>$ projection using a $300-\mathrm{kV}$ VG Microscopes HB603U STEM with Nion aberration corrector. (a) An incoherent angular dark-field image in which the Sr columns are the brightest features, the $\mathrm{Ti}$ columns less bright, and the $\mathrm{Al}$ and $\mathrm{O}$ columns are barely visible, while the (b) phase contrast bright-field image shows the Al and $\mathrm{O}$ columns with high contrast.
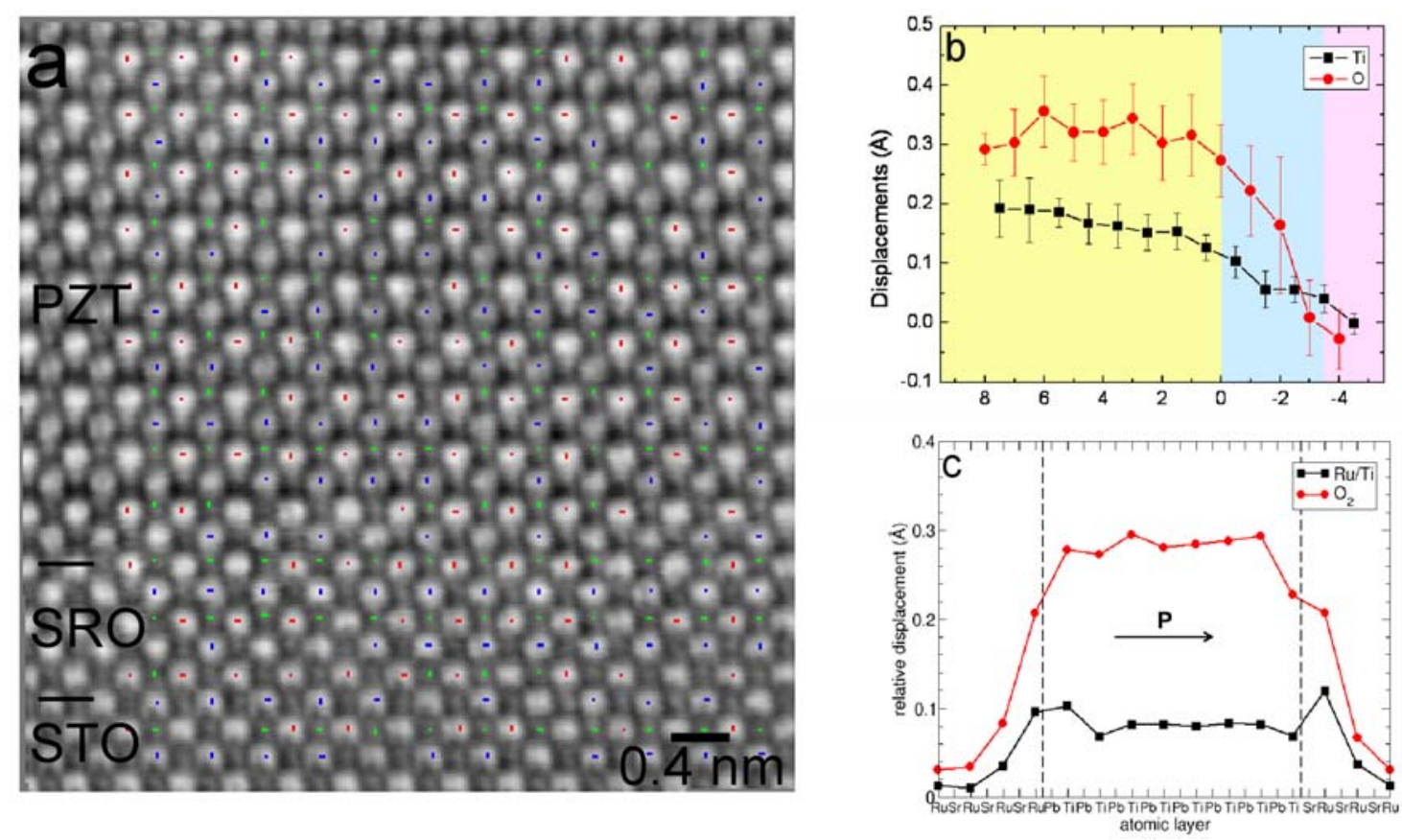

FIG. 2 Polar distortions in the vicinity of a PZT/SrRuO $3 / \mathrm{SrTiO}_{3}$ interface. (a) phase contrast bright-field image of the interface in the $<100>$ projection using a 300-kV VG Microscopes HB603U STEM with Nion aberration corrector. (b) Measured cation, oxygen distortions. (c) Calculated $\mathrm{Ti}$ and $\mathrm{O}$ distortions using a $\mathrm{PbTiO}_{3} / \mathrm{SrRuO}_{3}$ supercell. 